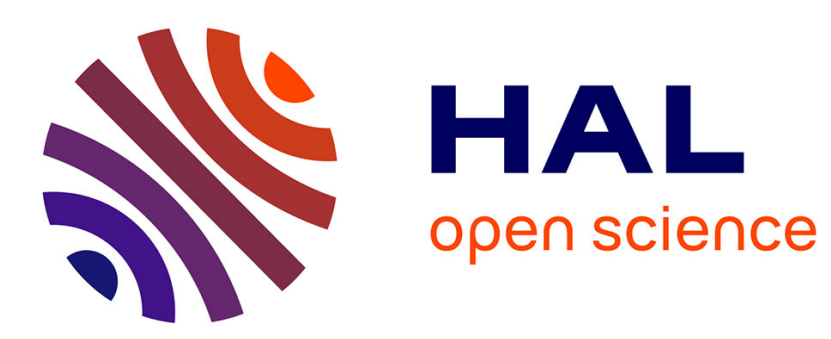

\title{
InAs QDs on InP : Polarization insensitive SOA and non-radiative Auger processes
}

Jacky Even, Laurent Pedesseau, François Doré, Soline Richard

\section{To cite this version:}

Jacky Even, Laurent Pedesseau, François Doré, Soline Richard. InAs QDs on InP: Polarization insensitive SOA and non-radiative Auger processes. Optical and Quantum Electronics, 2008, 40 (1415), pp.1233. 10.1007/s11082-008-9258-x . hal-00492357

\section{HAL Id: hal-00492357 https://hal.science/hal-00492357}

Submitted on 15 Jun 2010

HAL is a multi-disciplinary open access archive for the deposit and dissemination of scientific research documents, whether they are published or not. The documents may come from teaching and research institutions in France or abroad, or from public or private research centers.
L'archive ouverte pluridisciplinaire HAL, est destinée au dépôt et à la diffusion de documents scientifiques de niveau recherche, publiés ou non, émanant des établissements d'enseignement et de recherche français ou étrangers, des laboratoires publics ou privés. 


\title{
InAs QDs on InP : Polarization insensitive SOA and non-radiative Auger processes
}

\author{
J. Even*, L. Pedesseau, F. Dore and S. Boyer-Richard \\ FOTON UMR6082 CNRS, INSA Rennes, Campus Beaulieu,35042 Rennes Cedex, \\ France \\ (*author for correspondence: E-mail: jacky.even@insa-rennes.fr
}

\begin{abstract}
An efficient mechanical and electronic axial approximation of the strained $8 \times 8$ Hamiltonian is proposed for zinc-blende nanostructures with a cylindrical shape on (100) substrates. Vertically stacked InAs/InP columnar quantum dots (CQDs) for polarization insensitive semiconductor optical amplifier (SOA) in telecommunications applications are studied theoretically. Non-radiative Auger processes in InAs/InP quantum dots (QDs) are also investigated. It is shown that a multiband approach is necessary in both cases.
\end{abstract}

Key words: Quantum dot, $8 x 8$ k.p method, optical amplifier, Auger processes

\section{Introduction}

With a model recently proposed (Even et al. 2007, Even et al. 2008), it is possible to simulate large quantum dots (QD) systems using conventional finite element computation. The feasibility to describe complex inhomogeneous strain distribution and electronic properties of InAs/InP QD have been demonstrated by using cylindrical coordinates $(r, z)$. Recently, vertically stacked QD with a very small or zero spacing (columnar QD, CQD) have been investigated in order to obtain polarization insensitive semiconductor amplifiers (SOA). A crossover from a dominant TE optical ground state absorption to a TM dominant absorption is predicted for a number of layers equal to about 9 in good agreement with the experiment. It is associated to a clear symmetry change of the valence band (VB) electronic states. A model is also proposed to simulate various Auger processes involving carriers in the QD and in the surrounding barrier material.

\section{Axial approximation for the electronic properties of semiconductor QD}

The 8x8 QD Hamiltonian can be block diagonalized with a few approximations in a $F_{z}$ basis, where $F_{z}=J_{z}+L_{z}$ is the total angular momentum (Even et al. 2007, Even et al. 2008). The basis is constructed in a product form $\left|J, J_{z}\right\rangle\left|L_{z}=F_{z}-J_{z}\right\rangle$ where the first factor corresponds to one of the 8 band-edge Bloch functions related to the conduction band (CB), heavy holes (HH), light holes ( $\mathrm{LH})$ and 
split-off bands (SO) of the bulk material. The second factor $\left|L_{z}=F_{z}-J_{z}\right\rangle=\exp \left(i L_{z} \varphi\right) f(r, z)$ corresponds to the envelope functions adapted to the usual 1-band cylindrical representation ( $\mathrm{C}_{\infty \mathrm{v}}$ symmetry). $L_{z}=0, \pm 1, \pm 2, \ldots$ are related to the so-called S, P, D... radial functions of this representation. As a result, each electronic state in an axially QD is associated to a single value of the $F_{z}$ quantum number and is described by a sum of 8 products: $\Psi=\sum_{j=1-8}\left|J^{j}, J_{z}^{j}\right\rangle\left|L_{z}^{j}=F_{z}-J_{z}^{j}\right\rangle$ where the first terms correspond to the band-edge Bloch functions and the second terms to the envelope functions $\left|L_{z}^{j}\right\rangle=\exp \left(i L_{z}^{j} \varphi\right) f_{j}(r, z)$. A first approximation used for the $R$ term, is similar to the one for the block-diagonalization of the $4 \times 4$ or $6 \times 6 \mathrm{QW}$ Hamiltonian : $R=-\sqrt{3} \frac{\hbar^{2}}{2 m_{0}}\left[\gamma_{2}\left(k_{x}^{2}-k_{y}^{2}\right)-2 i \gamma_{3} k_{x} k_{y}\right] \approx-\sqrt{3} \frac{\hbar^{2}}{2 m_{0}} \bar{\gamma} k_{t}^{2} e^{-2 i \varphi} \cdot \gamma_{2}$ and $\gamma_{3}$ are replaced by a mean value $\bar{\gamma}=\frac{\gamma_{2}+\gamma_{3}}{2}$. In addition if the strain field is calculated using a continuum mechanical model (elasticity), we propose an axial approximation (symmetry reduction from $\mathrm{C}_{4 \mathrm{v}}$ to $\mathrm{C}_{\infty \mathrm{v}}$ ) which consists in defining of an effective modulus $\bar{C}$. The four non-zero strain components in cylindrical coordinates are calculated from the radial $u$ and vertical $w$ displacements: $\varepsilon_{r r}=\frac{\partial u}{\partial r}, \varepsilon_{\varphi \varphi}=\frac{u}{r}, \varepsilon_{z z}=\frac{\partial w}{\partial z}$ and $\varepsilon_{r z}=\frac{1}{2}\left(\frac{\partial u}{\partial z}+\frac{\partial w}{\partial r}\right)$.

Finally, for the strained part of the $8 \times 8$ bulk Hamiltonian, it is then possible to propose an additional axial approximation. It is applied to the $R_{\varepsilon}$ term by analogy to the unstrained Hamiltonian: $R_{\varepsilon}=\frac{b \sqrt{3}}{2}\left(\varepsilon_{x x}-\varepsilon_{y y}\right)-i d \varepsilon_{x y} \approx \frac{\bar{b} \sqrt{3}}{2}\left(\varepsilon_{r r}-\varepsilon_{\varphi \varphi}\right) e^{-i 2 \varphi}$. The coefficients containing the shear deformation potentials $b$ and $d$ are replaced by a mean value $\bar{b}: \frac{\bar{b} \sqrt{3}}{2}=\frac{1}{2}\left(\frac{b \sqrt{3}}{2}+\frac{d}{2}\right)$. Using this method we succeeded in recovering most of the results of much more time consuming full 3D calculations for InAs/InP QD (Cornet et al. 2006, Even et al. 2008). Clear selection rules are also obtained for the calculations of absorption spectra. In-plane polarized TE absorption is associated to transitions between two electronic states ( $F_{z 1}$ and $F_{z 2}$ ) with $\Delta F=\left|F_{z 1}-F_{z 2}\right|= \pm 1$ whereas for a TM absorption, $\Delta F=\left|F_{z 1}-F_{z 2}\right|=0$ (there is 
no absorption for other values of $\Delta F$ ). For a classical InAs/InP QD emitting at about $1.55 \mu \mathrm{m}$, the electronic and hole ground states (GS) correspond to $F_{z}= \pm 1 / 2$ and $F_{z}= \pm 3 / 2$ respectively. We can therefore conclude that an optical transition between these GS is only of TE type. Moreover, this method can be extended to include more than 8 bands (Richard et al. 2005).

\section{Columnar QD in polarization insensitive SOA}

Structures containing CQDs have been recently proposed in order to reach polarization insensibility in SOA (Kita et al. 2003, Kawagushi et al. 2006, Saito et al. 2008). We now examine InAs CQD geometries emitting at about $1.55 \mu \mathrm{m}$ as shown by the insert of figure 1-a for 7 layers of truncated cones. The chosen dimensions are $1.2 \mathrm{~nm}$ for the truncated cone height and $7 \mathrm{~nm}$ for the radius. The CQD are embedded into an $\operatorname{In}_{0.85} \mathrm{Ga}_{0.15} \mathrm{As}_{0.33} \mathrm{P}_{0.67}$ lattice-matched quaternary alloy. Kawagushi et al. 2006 have used an $\mathrm{In}_{0.66} \mathrm{Ga}_{0.34} \mathrm{As}_{0.44} \mathrm{P}_{0.56}$ tensile strain (1\%) quaternary alloy between the layers for strain compensation. The energy variations of the electronic degenerate GS and excited states are represented as a function of the number of layers on figure 1-b.
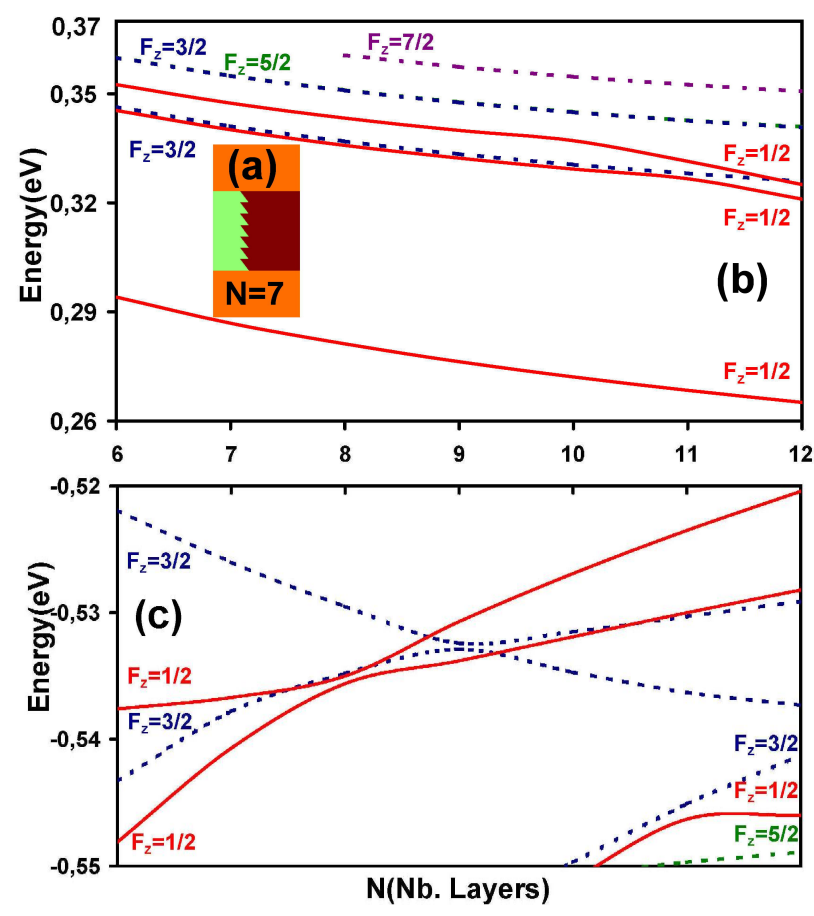

Fig. 1. (a) InAs CQD geometry corresponding to $N=7$ layers of truncated cones. (b) Dependence of the electronic conduction and (c) valence bands versus the number of layers. 
The electronic GS of the CQD corresponds to $\boldsymbol{F}_{z}= \pm 1 / 2$ whatever the number of layers like in the case of a single QD. The most striking feature appears for the hole states behavior (figure 1-c). A crossover between $F_{z}= \pm 3 / 2$ and $F_{z}= \pm 1 / 2$ for the hole GS is predicted for a number of layers close to 9 . Figure 2 is a representation for various numbers of layers of the transition matrix elements $M_{i f}=|\langle i|\hat{e} \cdot \hat{p}| f\rangle|^{2}$ for TE $(\hat{e} \perp o z)$ and TM $(\hat{e} / / o z)$ polarizations. All the optical transitions between monoelectronic states in the CQD are considered and the momentum matrix reads $\langle i|\hat{p}| f\rangle=\frac{m}{\hbar} \vec{\nabla}_{k_{\varepsilon}}\left\langle i \mid H_{k \cdot p}\left(\overrightarrow{k_{\varepsilon}}, \varepsilon\right) f\right\rangle$ with $k_{\varepsilon, i}=k_{i}-\sum_{j} \varepsilon_{i j} k_{j}$ (Enders et al. 1995). For $\mathrm{N}<9$, the absorption spectrum is predominantly $\mathrm{TE}$ at the ground state optical transition and TM at the first excited state optical transition. For $\mathrm{N}>9$, the absorption spectrum at the ground state optical transition is TE and TM with a predominant TM character. The interpretation proposed for InAs/GaAs CQD (Kita et al. 2003, Saito et al. 2008), is that the weight of the LH component in the hole GS becomes important as the number of stacks is increased. More precisely, our interpretation is that polarization insensitivity is obtained by a symmetry change of the hole GS, directly related to the change of the CQD aspect ratio (a CQD with a large number of stacks is similar to a quantum wire). Strain effects play a minor role whereas in the QW-based devices, symmetry change of the hole GS and polarization insensitivity are obtained with tensile strained alloys.

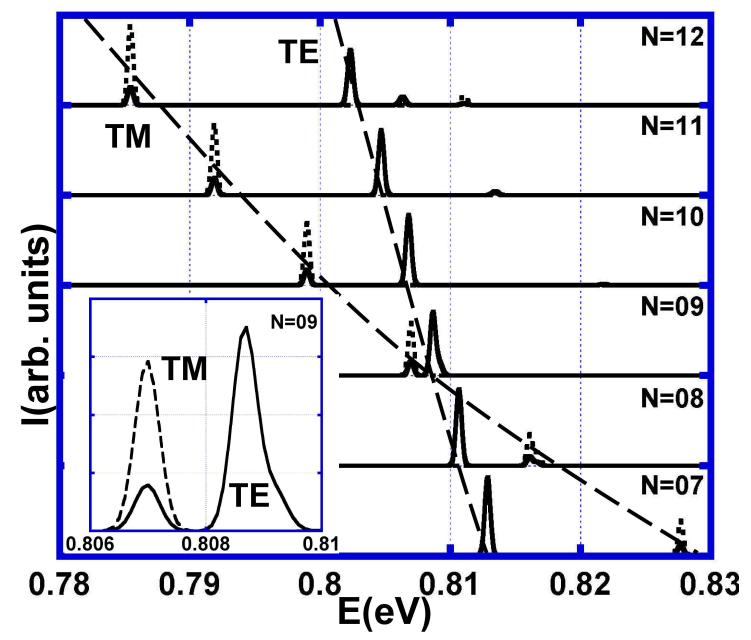

Fig. 2. TE and TM transition matrix elements $M_{\text {if }}$ of the optical transitions in a CQD for various numbers of layers 


\section{Non-radiative Auger processes in QD}

An InAs /InP QD laser may exhibit good properties such as low threshold current (Homeyer et al. 2007) but it has been shown experimentally that various Auger processes affect its dynamics. A first category of intradot Auger processes, involves the relaxation of a carrier either into the conduction band or into the valence band of the QD. In that case, the Auger process has a positive impact on the device performance (Veselinov et al. 2007, Miska et al. 2008). Most theoretical models published up to now consider the relaxation into the conduction band of the QD or neglect the influence of strain or VB coupling (Ferreira and Bastard 1999, Magnusdottir et al. 2003, Nielsen et al 2005, Even et al. 2007). In bulk materials or QW systems, Auger processes usually refer to nonradiative processes affecting interband optical transitions (Beattie and Landsberg 1958). This second category of Auger processes is almost not described in theoretical papers for QDs. Some authors even claim that these processes are either forbidden or negligible in QD (Jian al 1992). Experimental studies show however clearly that they have a negative impact on InAs/InP QD devices (Massé et al. 2007). It is possible to use our $8 \times 8 \mathrm{k} . \mathrm{p}$ model to simulate both categories of Auger processes simultaneously and also benefit from the axial approximation to simplify the 12-fold integrals associated to the simulations of Auger processes. (Beattie and Landsberg 1958). We have chosen to simulate Auger processes involving one carrier inside the QD and another one in the surrounding bulk material. For the intradot carriers, various processes may be compared with the same model (figure 3-a). The first two letters describe the transition of the carrier in the QD. The most efficient channel for the non-radiative Auger process related to the GS transition $(\mathrm{CH})$ is associated to a $\mathrm{SO}-\mathrm{HH}$ type transition $(\mathrm{SH})$ of the second carrier in the bulk ( $\mathrm{CHSH}$ process with $\tau_{\mathrm{CHSH}}=14 \mathrm{~ns}$, figure $3-\mathrm{b}$ ). The dynamics of this Auger process depend on the energy of the transition but also very much on the shape and the size of the QD. The most important parameter is the overlap between the two electronic GS. 

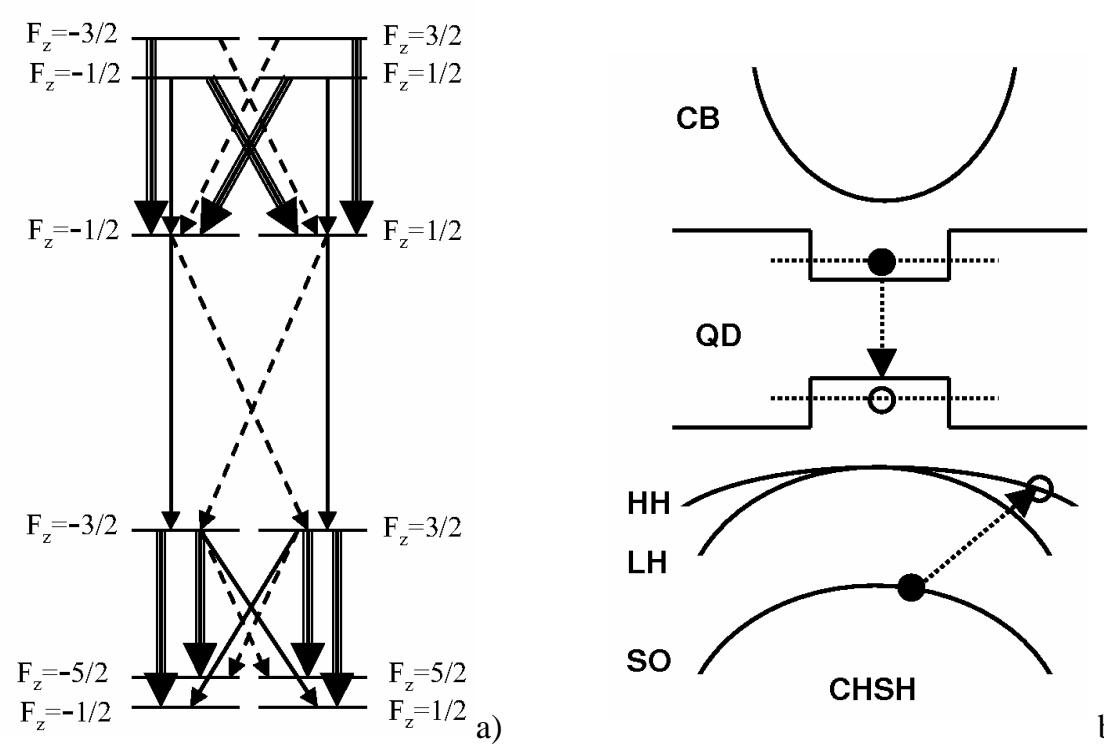

Fig. 3. a) Schematic representation of the non-radiative Auger processes in a conic InAs/InP QD. The ultrafast processes $(\sim$ ps $)$ are represented by big arrows with triple lines while fast $(\sim$ ns $)$ and slower $(<\mu \mathrm{s})$ are represented by arrows with full lines and dashed lines respectively. b) $\mathrm{CHSH}$ Auger process related to the GS transition $(\mathrm{CH})$ in a $\mathrm{QD}$ and to a $\mathrm{SH}$ (SO to $\mathrm{HH}$ ) type transition of the second carrier in the bulk

Ultrafast processes are associated to the relaxation of carriers into the electron (CC) or hole bands $(\mathrm{HH})$ of the QD. In that case a transition of the second carrier in the bulk $\mathrm{CB}$ (CC type) is the most efficient one. For example, if we consider an InAs truncated cone emitting at $0.77 \mathrm{eV}(\mathrm{T}=0 \mathrm{~K})$ in a $\mathrm{In}_{0.85} \mathrm{Ga}_{0.15} \mathrm{As}_{0.33} \mathrm{P}_{0.67}$ matrix, $\tau_{\mathrm{CCCC}}=0.7 \mathrm{ps}$ and $\tau_{\mathrm{HHCC}}=1 \mathrm{ps}$.

\section{Conclusion}

A new 8x8 k.p model with a few approximation is used to simulate polarization insensitivity in CQD and various Auger processes. It appears that the coupling between the electronic states must be described in details in the two cases, and that the various components of the wavefunctions must be included.

\section{Acknowledgments}

This work is supported by Sandie European Network of excellence and French ANR project

"Biquinis". 


\section{References}

Beattie A. R. and Landsberg P.T.: Auger effects in semiconductors, Proc. R. Soc. A 249, 16-29 (1958)

Cornet C., Schliwa A., Even J., Doré F., Celebi C., Letoublon A., Macé E., Paranthoen C., Simon A., Koenraad P.M., Bertru N., Bimberg D. and Loualiche S., : Electronic and optical properties of InAs/InP quantum dots on InP(100) and InP(311)B substrates: Theory and experiment, Phys. Rev. B 74, 035312 (2006)

Enders P., Bärwolff A.and Woermer M. : k.p theory of energy bands, wave functions, and optical selection rules in strained tetrahedral semiconductors, Phys. Rev. B 51, 16695-16704 (1995)

Even J., Doré F., Cornet C., Pédesseau L., Schliwa A. and Bimberg D.: Semianalytical evaluation of linear and nonlinear piezoelectric potentials for quantum nanostructures with axial symmetry, Appl. Phys. Lett. 91, 122112 (2007)

Even J., Cornet C. and Doré F.: Anisotropic and inhomogeneous Coulomb screening in the Thomas-Fermi approximation: Application to quantum dot-wetting layer system and Auger relaxation, Physica Status Solidi (b) 244, 3105-3114 (2007)

Even J., Doré F., Cornet C. and Pédesseau L.: Semianalytical model for simulation of electronic properties of narrow-gap strained semiconductor quantum nanostructures, Phys. Rev. B 77, 085305 (2008)

Ferreira R. and Bastard G.: Phonon-assisted capture and intradot Auger relaxation in quantum dots, Appl. Phys. Lett. 74, 2818-2820 (1999)

Homeyer E., Piron R., Grillot F., Dehaese O., Tavernier K., Macé E., Even J., Le Corre A. and Loualiche S.: Demonstration of a Low Threshold Current in 1.54 $\mu \mathrm{m}$ InAs/InP(311)B Quantum Dot Laser with Reduced Quantum Dot Stacks, Jap. J. Appl. Phys. 46, 6903-6905 (2007)

Jian Y., Teich M.C., Wang W.I. and Meyer J.R.: Auger recombination in HgCdTe quantum wires and quantum boxes, J. Appl. Phys. 71, 3394-3398 (1992)

Kawagushi K., Ekawa M., Yasuaka N., Akiyama T., Ebe H., Sugawara M. and Arakawa Y. : 1.3-1.6 $\mu \mathrm{m}$ broadband polarization-independent luminescence by columnar InAs quantum dots on InP(001), Phys. Stat. Sol. (c) 3, 3646-3651 (2006)

Kita K., Jayavel P., Wada O., Ebe H., Nakata Y. and Sugawara M.: Polarization controlled edge emission from columnar InAs/GaAs self-assembled quantum dots, Phys. Stat. Sol. (c) 0, $1137-$ $1140(2003)$

Magnusdottir I., Bischoff S., Uskov A.V. and Mork J.: Geometry dependence of Auger carrier capture rates into cone-shaped self-assembled quantum dots, Phys. Rev. B 67, 205326 (2003) Massé N.F., Homeyer E., Marko I.P., Adams, A.R., Sweeney S.J., Dehaese O., Piron R., Grillot F. and Loualiche S.: Temperature and pressure dependence of the recombination processes in $1.5 \mu \mathrm{m}$ InAs/InP (311)B quantum dot lasers, Appl. Phys. Lett. 91, 131113 (2007)

Miska P., Even J., Dehaese O. and Marie X.: Carrier relaxation dynamics in InAs/InP quantum dots, Appl. Phys. Lett. 92, 191103 (2008)

Nielsen T.R., Gartner P. and Jahnke F.: Many-body theory of carrier capture and relaxation in semiconductor quantum-dot lasers, Phys. Rev. B 69, 235314 (2004) 
Richard S., Aniel F. and Fishman G.: Band diagrams of Si and Ge quantum wells via the 30-band k·p method, Phys. Rev. B 72, 245316 (2005)

Saito T. , Ebe H., Arakawa Y., Kakitsuka T. and Sugawara M.: Optical polarization in columnar InAs/GaAs quantum dots: 8-band k·p calculations, Phys.Rev. B 77, 195318 (2008)

Veselinov K., Grillot F., Cornet C., Even J., Bekiarski A., Gioannini M. and Loualiche S. : Analysis of the double laser emission occurring in 1.55- $\mu \mathrm{m}$ InAs-InP (113)B quantum-dot lasers.

IEEE J. Quantum Electron. 43(9), 810-816 (2007) 